\title{
MODULATION INSTABILITY AND CONSERVATION OF ENERGY: TOWARDS A NEW MODEL
}

\author{
Silvello Betti ${ }^{1}$, Elisa Duca ${ }^{1,2}$, Mauro Giaconi ${ }^{1}$, Giorgia Parca ${ }^{1,3}$ \\ ${ }^{1}$ Università degli Studi di Roma Tor Vergata, Via del Politecnico 1, 00133 Roma \\ betti@ing.uniroma2.it \\ ${ }^{2}$ Agenzia Spaziale Italiana, Viale Liegi 26, 00198 Roma \\ ${ }^{3}$ Instituto de Telecomunicações, Campus de Santiago, 3810-197, Aveiro, Portugal
}

Abstract - We investigate nonlinear a perturbed CW signal propagation. Studies in the literature define parameters for the interaction without indications about the boundaries of the model. Conservation-ofEnergy is considered as constraint to investigate the nonlinear regime

\section{Introduction}

Optical fiber, under proper conditions, may couple amplitude and phase of a single or a set of propagating signals (SPM - XPM). Moreover, nonlinearities may generate other fields at specific wavelengths starting from a comb of injected signals (FWM). Most of these impairments can be successfully exploited to obtain: pulse reshaping, soliton propagation, dispersion compensation or wavelength conversion. So, apparently disadvantageous phenomena may be transformed into powerful tools to carry out all-optical signal processing. To this aim, an accurate modeling of optical nonlinear propagation in fiber is mandatory. As it is well described in the literature [1-2], Modulation Instability (MI) is a nonlinear phenomenon triggered under proper conditions: a strong Continuous Wave signal (CW) propagates along an optical fiber together with a small perturbation (e.g., a spectral component of Amplified Spontaneous Emission - ASE - noise or Relative Intensity Noise - RIN). Under the hypothesis of undepleted pump, it is possible to describe the signal propagation by defining a spectral gain. Since the pump energy feeds the perturbation amplification, it is worth to introduce the Conservation of Energy in the analysis [3]. Thus, the model can be extended considering the pump depletion process. It leads to distinguish the very first region, in which the phenomenon is triggered, the pump is still considered undepleted and the perturbation is negligible, and a further region where the pump energy enhances the amplified perturbation, no longer constant along the fiber. The first region is well described in the literature, but a deeper analysis requires to model also the second one. In this paper we define the boundary between the two regions, by introducing an Effective Length. First section of this paper gives a description of the analytical model based on a novel approach. In the second section of the paper, the Conservation of Energy is introduced, in order to extend the model taking into account the overall optical power travelling along the fiber. In particular, through the Energy Conservation condition, we consider that the MI gain comes from the pump signal depletion. Thus, our model extension is based on the pump power dependence on distance. This leads to define a region where the well known results [1-2] are no longer valid. This novel approach introduces a parameter, the Effective Length, used to measure the distance beyond which the CW pump cannot be considered undepleted, and gain saturation is appreciable [3]. Conclusions and future works end the paper.

\section{Perturbative Approach}

A general expression for the complex envelope of a propagating signal is:

$A(z, t)=H(z, t) g / t(z, t)$

where $\mathrm{H}$ and $\psi$ are the field amplitude and phase, respectively. Starting from the Non-Linear Schrödinger Equation (NLSE) [1] we obtain the following equation for the complex envelope:

$$
a \frac{\partial H}{\partial z}+j \frac{\partial \psi}{\partial z} H+j \frac{\beta}{2} \frac{\partial^{2} H}{\partial t^{2}}-\beta \frac{\partial \psi}{\partial t} \frac{\partial H}{\partial t}-\frac{\beta}{2} \frac{\partial^{2} \psi}{\partial t^{2}} H-j \frac{\beta}{2}\left(\frac{\partial \psi_{t}}{\partial t}\right)^{2} H=j H^{3}-\frac{\alpha}{2} H
$$

where $\beta$ is the Group Velocity Dispersion (GVD) parameter, $y$ is the nonlinear coefficient, and $\alpha$ represents the fiber loss. This complex equation can be split into a system of two equations, one for the real and one for the imaginary component:

$$
\left\{\begin{array}{c}
\frac{\partial H}{\partial z}=\beta \frac{\partial H}{\partial t} \frac{\partial \psi}{\partial t}+\frac{\beta}{2} \frac{\partial^{2} \psi}{\partial t^{2}}-\frac{\alpha}{2} H \\
\frac{\partial \psi}{\partial z} H=\frac{\beta}{2}\left(\frac{\partial \psi}{\partial t}\right) H-\frac{\beta}{2} \frac{\partial^{2} H}{\partial t^{2}}+\gamma H^{3}
\end{array}\right.
$$


In our analysis we study a propagating signal expressed as a combination of a strong injected CW signal and a weak perturbation, in order to understand the system behavior in a perturbed-equilibrium state. The perturbation may be related, for instance, to amplifier noise [4-5] (ASE) or LASER noise (RIN). Thus, the global function becomes:

$$
A(z, t)=\mathbf{L} H(z, t)+h(z, t)] e^{i \varphi(z, t)}
$$

where both $h(t, t)$ and $\varphi(z, t)$ are real functions of time and space. Under the hypothesis of undepleted CW pump signal, one gets: $H(z, t)=H_{0}$ with $H_{0}=H(0, t)$. As soon as the conservation of energy will be introduced, this hypothesis shall be removed. By calculating the NLSE one gets:

$$
\left\{\begin{array}{c}
\frac{\partial \varphi}{\partial z}+\frac{\beta}{2\left(\mathrm{H}_{0}+h\right)} \frac{\partial^{2} h}{\partial t^{2}}-\frac{\beta}{2}\left(\frac{\partial \varphi}{\partial t}\right)^{2}=\gamma\left(H_{0}+h\right)^{2} \\
\frac{\partial h}{\partial z}-\frac{\beta}{2} \frac{\partial^{2} \varphi}{\partial t^{2}}\left(H_{0}+h\right)-\beta \frac{\partial h}{\partial t} \frac{\partial \varphi}{\partial t}=0
\end{array}\right.
$$

For simplification, we neglect the fiber loss, substantially irrelevant for the present analysis. Hereafter, we distinguish the analysis in case the ratio between $h$ and $\mathrm{H}_{0}$ is very small $(A)$, or gradually higher due to the growth of the initially weak perturbation (B). In order to quantify the ratio between $\mathrm{H}_{0}$ and $h$, we introduce the parameter $M=$ $\mathrm{H}_{0} / \mathrm{h}$.

\section{Approximation 1: Kerr Dominating, small perturbation}

We assume $M » 1\left(H_{0} » h\right)$ and a small phase dynamics. We neglect all polynomial terms higher than the first degree or both the perturbation amplitude $(h)$ and phase $(\varphi)$, as well as all terms proportional to the ratio $h / H_{0}$. Under these hypotheses, the set of equations can be rewritten as:

$$
\left\{\begin{array}{c}
\frac{\partial \varphi}{\partial z} \cong \gamma H_{0}^{2}+2 \gamma H_{0} h \\
\frac{\partial h}{\partial z} \cong \frac{\beta H_{0}}{2} \frac{\partial^{2} \varphi}{\partial t^{2}}
\end{array}\right.
$$

The phase variations in $z$ are due to Kerr effect, because of both the CW signal power and the beating term between the CW signal and the perturbation one. By a space derivative of the second equation, and using the first one, one gets:

$$
\frac{\partial^{2} h}{\delta z^{2}}=\gamma H_{0}^{2} \frac{\partial^{2} h}{\partial t^{2}}
$$

A linear dynamic system is considered, so its behavior is studied by applying sinusoidal signals with a generic angular frequency (harmonic analysis). So doing, the general harmonic solution for this differential linear equation is given by:

$$
h(z, t)=h_{0} e^{\sqrt{-\gamma \beta} H_{\mathrm{g}} \Omega z} e^{-i n t}+c . c .
$$

being c.c. the complex conjugate term, and $\widetilde{\boldsymbol{h}}_{0}$ the initial perturbation amplitude. The term in the square root is positive only if $\beta<0$. So, this parameter defines the instable regime when $\Omega_{1}(\Omega)=F_{0} \Omega_{\sqrt{-\gamma \beta}}$ is real. This result confirms the instable regime depends on the $\operatorname{sign}$ of $\beta$, but it does not give any information about the influence of angular frequency of the perturbation itself. In the following sub-section, it is shown how this information comes from a more accurate analysis.

\section{Approximation 2 : A further step in the analysis}

After the triggering of the phenomenon, the initial approximation, in which the perturbation is small, is slightly modified and the ratio $h / \mathrm{H}_{0}$ is assumed not infinitesimal anymore, i.e. all proportional terms are taken into account. By following the same approach as in the previous sub-section, we get:

$$
\frac{\partial^{2} h}{\partial z^{2}}=-\frac{\beta^{2}}{4} \frac{\partial^{4} h}{\partial t^{4}}+\gamma \beta H_{0}^{2} \frac{\partial^{2} h}{\partial t^{2}}
$$

Since the Kerr gives a constant contribution to the phase, it does not participate in Eq.10. We choose the same formalism as in Eq.8, thus:

$$
h(z, t)=\hbar(z) e^{j n t}+6 . G .
$$

By substituting Eq. 10 into Eq. 9, one gets:

$$
\frac{\partial^{2} h}{\partial z^{2}}=\left(-\frac{\beta^{2}}{4} \Omega^{4}-\gamma \beta \mathrm{H}_{0}^{2} \Omega^{2}\right) h
$$

Also in this case, we find two possible operative regions: the stable and the instable regime. We observe that the boundary definition is slightly different from the previous one. In particular, we get $g_{2}(\Omega)$, as:

$$
B_{2}(\Omega)=\Omega \sqrt{\left(-\frac{\beta^{2}}{4} \Omega^{2}-\gamma \beta \mathrm{H}_{0}^{2}\right)}
$$


The perturbation grows exponentially when $\varepsilon_{2}(\Omega)$ is a real number. Thus, the conditions for instable regime depends also on the spectral position of the perturbing signal with respect to the pump signal. If we introduce the threshold angular frequency $\Omega_{\mathrm{t}}=\sqrt{-\frac{4 \gamma R_{0}^{2}}{\beta}}$, which gives instable solutions only in case $\beta<0$.

Only signals with angular frequency lower than the threshold grow exponentially while propagating along the fiber with a gain $\theta_{2}(C)$. The gain expression is the same as in [1]. It straightforward that if the perturbation experiences a gain, there exists a distance from which the previous perturbative approach is no longer applicable. In order to put the basis for the model extension, in the following we introduce the condition of the conservation of energy, which relates the gain experienced by the perturbation to the pump depletion.

\section{Conservation of Energy}

So far we described an approach to find out the operative conditions, in terms of fiber and signal parameters, which may trigger MI. From what we obtained, it is reasonable to expect that the $\mathrm{CW}$ acts as a pump and it undergoes a depletion along its propagation. Thus, the initial condition of undepleted CW pump should be removed as soon as the perturbation is transformed into a non negligible signal. If the attenuation is not taken into account, as we assumed in the previous section, the overall optical power must be constant:

$$
\int_{i}^{i+T} \mid A(z, t) \boldsymbol{\Gamma}^{2} a t=K
$$

where $A(z, t)$ is the field in Eq.1. During its propagation, the optical power calculated over a proper time interval $T$ shall be constant with respect to $z$ :

$$
\frac{\partial}{\partial z} \int_{\tau}^{\tau+T}[H(z, t)+h(z, t)]^{2} d t=0
$$

The pump depletion can now be calculated. From previous section we get a simple expression for the perturbation, deriving from the initially undepleted pump. As first step, we consider the same perturbation behavior, thus, the integration time is set according to the angular frequency $(T=1 / \Omega)$. The resulting expression, is [3]:

$$
\tilde{h}(z) \frac{\partial h^{\prime}}{\partial z}-2 \tilde{H}_{0}(z) \frac{\partial F_{0}}{\partial z}
$$

where tilde notation represents the space dependent field amplitude. This result confirms that the spatial variation of $\tilde{H}_{0}(\mathrm{Z})$ is negligible only if the hypothesis of very high $\mathrm{M}$ (used in I-A and I-B) is valid.

\section{Effective Length and pump depletion}

We start from this point to investigate the limit for applying the perturbative approach. Since the perturbation $\tilde{h}(z)$ experiences a gain, the CW signal shall undergo depletion as suggested by Eq. 15 [6]. We introduce the effective length $\left(L_{e}\right)$ corresponding to a reduction by a factor $1 / \mathrm{e}$ of the initial $\mathrm{CW}$ signal:

$$
H\left(L_{\sigma}\right)=\frac{H_{0}}{e} \text {. }
$$

Assuming the expression found for $\tilde{h}(z)$ to be conservative, the effective length can be written as:

$$
L_{\Theta}=\frac{1}{2 g_{2}(\Omega)} \ln \left[2 \frac{B_{0}^{2}}{h_{0}^{2}}\left(1-\frac{1}{\theta}\right)+1\right]
$$

with the gain $\mathrm{g}_{2}$ given by Eq.12.

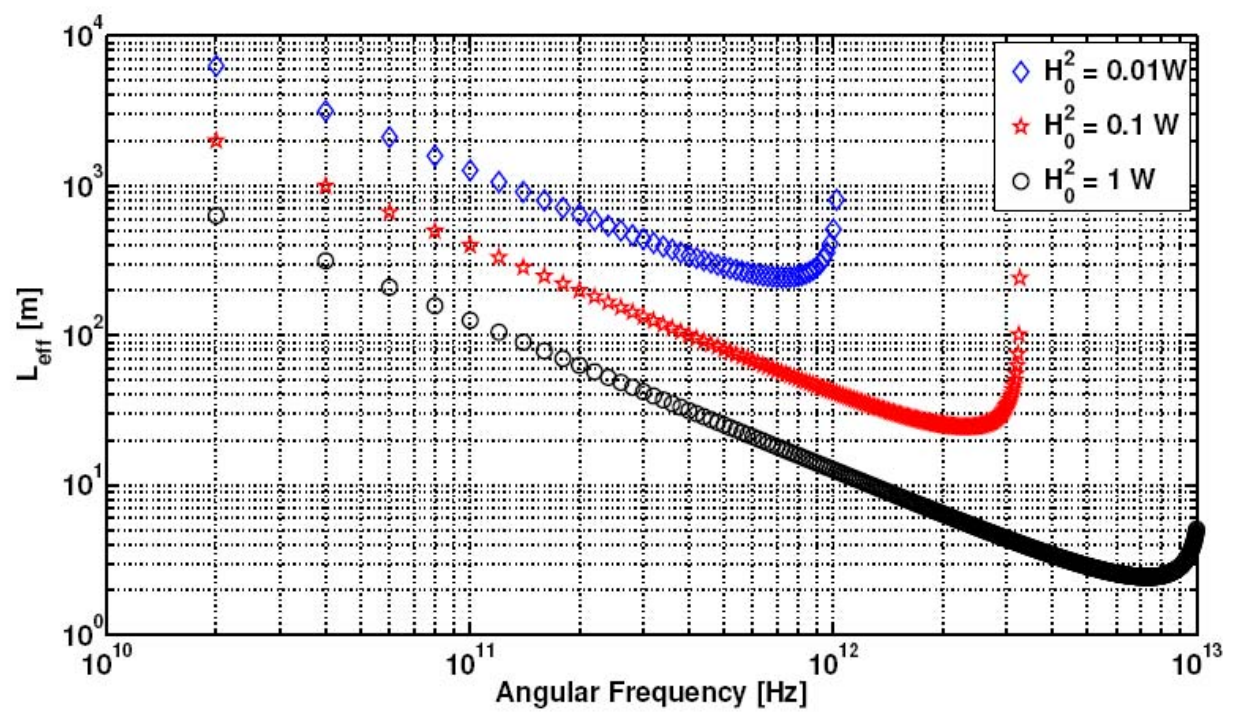

Figure 1 Effective length defines a boundary for the perturbative approach applicability 
In Figure 2 we show the Effective Length as a function of the angular frequency for three input power levels ( $\left.\mathcal{K}_{0}^{2}\right)$. When the perturbation is spectrally far from the CW $\left(|\Omega| » \Omega_{t}\right)$ the instability is not triggered and the CW remains an undepleted pump. On the other hand, any weak signal laying within the gain bandwith, is amplified. In fact, the pump depletion is a consequence of a dispersion-related phenomenon which distributes optical power over a wider spectrum. A simple experiment shows how the pump is depleted generating a wideband gain experienced by a weak perturbation. The pump depletion can be easily measured using the same scheme as described in [3]. A narrowband laser sources feeds an optical amplifier, whose ASE acts as perturbation source, and the signal is injected into a NZD fiber. The output Power is sent to an OSA. By varying the injected optical power level, the measured output optical power at OSA level does not linearly follow the same trend. The measurement was carried out on a NZD fiber with GVD=2.2 ps/km/nm (Dispersion Coefficient) and $\alpha=0.26 \mathrm{~dB} / \mathrm{km}$ (Fiber Attenuation).

The power transfer function can be derived by:

$$
|H(f)|^{2}=\frac{P_{0 w i}(f)}{P_{t n}(f)}
$$

where $F_{\text {in }}(f)$ and $F_{\text {sit }}(f)$ are the Power Spectral Densities at the input and output of the fiber, respectively. The input PSD was measured by the OSA fed by the optical signal injected into the $50 \mathrm{~km}$ long fibre, and the output PSD was measured by the OSA collecting the optical signal after propagating along $50 \mathrm{~km}$ long fibre. As it is shown in Figure 3 , on the one hand the $\mathrm{CW}$ undergoes a strong power reduction, on the other hand there are sidebands where a positive net gain is measurable, including the attenuation of about $10 \mathrm{~dB}$ over the fiber length.

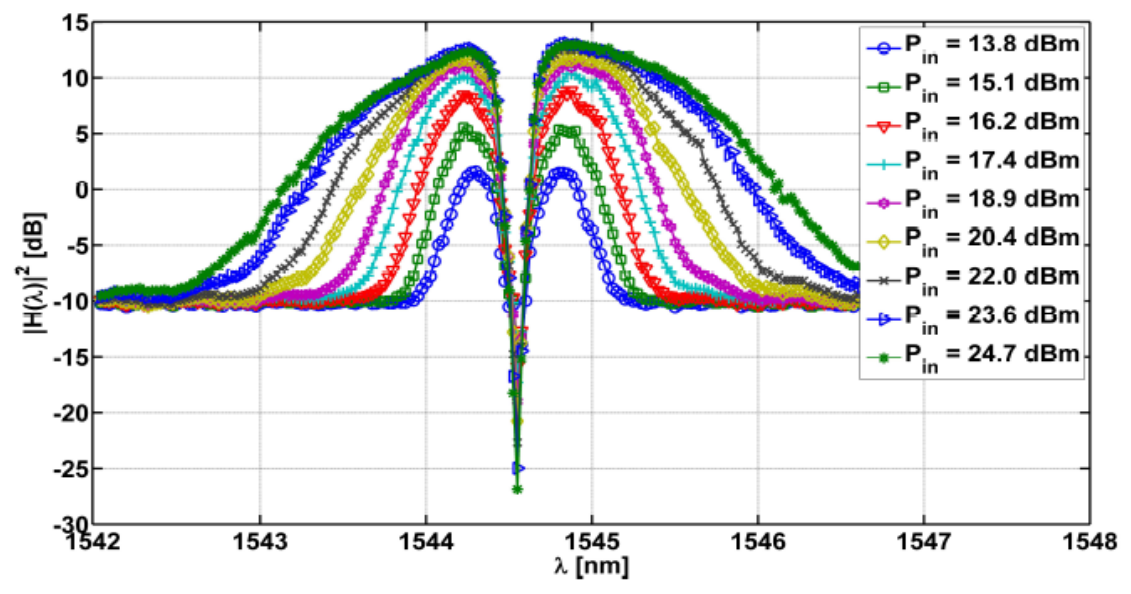

Figure 3 Power transfer function for $50 \mathrm{~km}$ dispersive fiber.

\section{Conclusion}

In this paper we define the boundary conditions for MI modeling based on small perturbation. Thanks to the introduction of the Conservation of Energy, the Effective Length limits the hypothesis of undepleted pump within a region, where the well-known results from the literature are applicable.

Beyond that boundary, a new model is required, taking into account the energy is transferred from pump signal to sidebands by means of the fiber parameters.

\section{References}

[1] G. Agrawal, Nonlinear fiber optics, ser. Optics and Photonics. San Diego, CA USA: Academic Press, 2001.

[2] M. Potasek and G. Agrawal, "Self-amplitude-modulation of optical pulses in nonlinear dispersive fibers," Phisycal Review A, vol. 36, no. 8, Oct. 1987.

[3] E. Duca, M. Giaconi, G. Parca, "Pump depletion measurement under Modulation Instability conditions", $12^{\circ}$ International Conference on Transparent Optical Networks 2010, (ICTON 2010) June 2010, doi: 10.1109/ICTON.2010.5549112.

[4] S. Sastry, Nonlinear System Analysis, Stability and Control. New York, NY USA: Springer Verlag, 1999.

[5] C. Lorattanasane and K. Kikuchi, "Parametric instability of optical amplifier noise in long-distance optical transmission systems," IEEE Journal of Quantum Electronics, vol. 33, no. 7, pp. 1068-1074, Jul. 1997. 